\title{
Antibiotic perturbation of mixed-strain Pseudomonas aeruginosa infection in patients with cystic fibrosis
}

\author{
Anna S. Tai ${ }^{1,2,3^{*}}$ D, Laura J. Sherrard ${ }^{4 \dagger}$, Timothy J. Kidd ${ }^{5,6,7}$, Kay A. Ramsay ${ }^{1,4}$, Cameron Buckley ${ }^{8}$, Melanie Syrmis ${ }^{8,9}$, \\ Keith Grimwood ${ }^{7,10}$, Scott C. Bell ${ }^{2,4}$ and David M. Whiley 8,9
}

\begin{abstract}
Background: Pulmonary exacerbations in cystic fibrosis (CF) remain poorly understood and treatment is usually targeted at Pseudomonas aeruginosa. Within Australia a predominant shared P. aeruginosa strain (AUST-02) is associated with greater treatment needs. This single centre study assessed temporal shared strain population dynamics during and after antibiotic treatment of exacerbations.

Methods: Sputum was collected from 12 adult patients with a history of chronic AUST-02 infection at four time-points during and after treatment of an exacerbation. Forty-eight $P$. aeruginosa isolates within each sample underwent AUST-02 allele-specific PCR and SNP-based strain genotyping.

Results: Various commonly shared Australian strains (AUST-01, 0.1\%; AUST-02, 54.3\%; AUST-06, 36.6\%; AUST-07, 4.6\%; AUST-11, 4.3\%) and two unique strains (0.1\%) were identified from 45 sputum samples (2160 isolates). Based on within-patient relative abundance of strains, a "single-strain infection" $(n=7)$ or "mixed-strain infection" $(n=5)$ was assigned to each patient. A significant temporal variation in the $P$. aeruginosa population composition was found for those with mixed-strain infection $(P<0.001)$. Patients with mixed-strain infections had more long-term treatment requirements than those with single-strain infection. Moreover, despite both groups having similar lung function at study entry, patients with single-strain infection had greater improvement in $\mathrm{FEV}_{1} \%$ predicted following their exacerbation treatment $(P=0.02)$.
\end{abstract}

Conclusion: Pulmonary exacerbations may reveal multiple, unrelated $P$. aeruginosa strains whose relative abundance with one another may change rapidly, in a sustained and unpredictable manner.

Keywords: Cross-infection, Cystic fibrosis, Disease progression, Pseudomonas Aeruginosa, Respiratory tract infections

\section{Background}

Despite improved survival for people with cystic fibrosis (CF) [1], most still die prematurely from chronic pulmonary infections characterised by recurrent exacerbations, progressive lung function decline, increased treatment requirements and reduced quality of life [2-6]. The pathophysiology of pulmonary exacerbations is nevertheless poorly understood and a standardised definition of an exacerbation remains elusive [7-9].

\footnotetext{
* Correspondence: sze.tai@health.wa.gov.au

${ }^{\dagger}$ Equal contributors

${ }^{1}$ School of Medicine, The University of Queensland, Brisbane, QLD, Australia ${ }^{2}$ Adult Cystic Fibrosis Centre, Department of Thoracic Medicine, The Prince Charles Hospital, Brisbane, QLD, Australia

Full list of author information is available at the end of the article
}

Pseudomonas aeruginosa is the most common pulmonary pathogen in $\mathrm{CF}$ and antibiotic treatment of exacerbations directed against this organism is pivotal to patient management [10]. Once $P$. aeruginosa becomes established within the airways of patients with chronic lung disease, it is usually by a single strain that evolves through micro-adaptation into multiple sub-lineages of the original ancestral clone [11, 12]. There are however, reports of co-infection with two or more distinct $P$. aeruginosa genotypes in both $\mathrm{CF}$ [13-15] and, non-CF bronchiectasis [12]. In Australia, a predominant shared strain, AUST-02, was detected in $18 \%$ of all patients with $P$. aeruginosa infection nationally and was associated with increased centre visits and intravenous antibiotic 
courses [13]. Given the high-prevalence and clinical significance of the AUST-02 strain within Australian centres, including its predominance in our own clinic, the primary aim of this study was to assess AUST-02 population stability and determine whether other $P$. aeruginosa strains emerged during and after treating an exacerbation.

\section{Methods}

\section{Study patients and characteristics}

Twelve CF patients (aged $\geq 18$-years) with chronic AUST02 infection, defined according to the modified Leeds Criteria $[16,17]$, were recruited over a 5 -month period between February and June 2014 following admission to The Prince Charles Hospital (Brisbane, Australia) for intravenous antibiotic treatment of an exacerbation (as defined by the Fuchs criteria [9]). While in hospital, CF patients with $P$. aeruginosa were not managed exclusively in single room accommodation with some admitted to shared rooms [18]. The Prince Charles Hospital Human and Research Ethics Committee, Metro North Hospital and Health Service, Brisbane, Queensland, Australia approved the study (HREC/13/QPCH/127) and all participants provided written, informed consent.

Baseline characteristics of age, gender, cystic fibrosis transmembrane conductance regulator (CFTR) genotype, and the best lung function (forced expiratory volume in the first second percentage predicted; $\mathrm{FEV}_{1} \%$ predicted [19]) and body mass index (BMI) recorded in the previous year were collected from hospital records. Years chronically infected by $P$. aeruginosa, clinical care requirements in the previous year (number of hospital admissions, inpatient days and outpatient clinic visits), types of treatments in the previous year (oral azithromycin, inhaled tobramycin, inhaled colistin) and diagnoses of diabetes and liver disease were also recorded. Clinical data collected during their hospitalisation included $\mathrm{FEV}_{1} \%$ predicted and $\mathrm{BMI}$ at the start-of-treatment and end-of-treatment time-points. Serum C-reactive protein (CRP) was measured on days 3 and 10 of inpatient stay, according to a local treatment protocol (for consistency hereafter referred to as start-of-treatment and end-oftreatment). Types of antibiotics administered, antibiotic duration, relapse (defined as readmission to hospital with a further pulmonary exacerbation before outpatient review occurred) and time-to-next hospitalisation for an exacerbation were collated.

\section{Sputum collection, culture and genotyping}

Spontaneously expectorated sputum was collected at study entry when commencing intravenous antibiotics ('start-of-treatment'), during the first-week of treatment ('during treatment'), at its completion ('end-of-treatment') and during outpatient review following discharge or if readmission to hospital before outpatient review occurred ('follow-up').

Sputum samples were processed as described previously to isolate single colonies [20]. Quantitative cultures were performed using standard techniques. A sweep of colonies from the 'start-of-treatment' sputum samples was tested for AUST-02 by an allele-specific polymerase chain reaction (PCR; Additional file 1: Supplementary Methods). In addition, 48 individual presumptive $P$. aeruginosa colonies were selected randomly for genotyping from each sputum sample the 12 study patients provided.

Bacterial DNA preparation was undertaken by heatdenaturation [21]. Each isolate underwent testing by the above AUST-02 allele-specific PCR (Fig. 1 and Additional file 1: Supplementary Methods) with isolates testing negative for AUST-02 subjected to single nucleotide polymorphism (SNP)-based strain typing (Agena; formerly Sequenom iPLEX) at the Australian Genome Research Facility (AGRF, The University of Queensland, Brisbane) based on a protocol described previously [22].

\section{Phenotypic testing}

The first recruited subject had a mixed-strain infection and each $P$. aeruginosa isolate genotyped from this patient underwent additional phenotypic testing to determine the within-host diversity. This sub-analysis involved 186 isolates with a total of 2790 phenotypic tests completed. Susceptibility to 11 anti-pseudomonal antibiotics was determined by disc diffusion [23], auxotrophy by growth on M9 minimal media [11] and alginate overproduction by the appearance of mucoid isolates on Pseudomonas Isolation Agar after overnight aerobic incubation at $36{ }^{\circ} \mathrm{C}$ [24]. Inactivating lasR mutant colonies were identified by their metallic iridescent sheen on Luria Bertani agar following overnight incubation in aerobic conditions at $36{ }^{\circ} \mathrm{C}$ [25], while pyocyanin production was quantified by measuring the $A_{695}$ value as described previously [11].

\section{Statistical analyses}

A repeated measures analysis of variance (ANOVA) was used to examine if the log-transformed $P$. aeruginosa total viable count differed between time-points of sputum collection. Clinical variables were compared between time-points or groups using a paired t-test (or Wilcoxon signed-rank test), independent $t$-test (or Mann-Whitney test) or Fisher's exact test, as appropriate. Variation in the number of strains detected and phenotypic traits were assessed by chi-square tests. All statistical analyses were generated using SPSS version 22. A two-tailed, $P<0.05$ was considered statistically significant. 


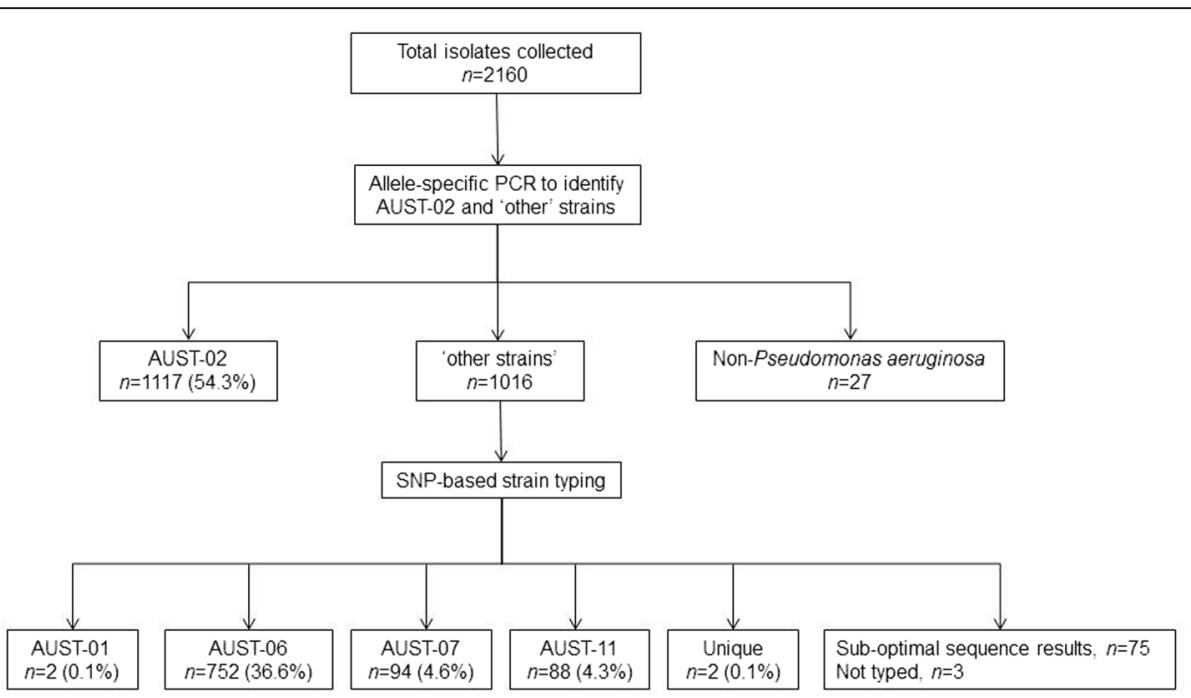

Fig. 1 Number of isolates randomly selected from 45 sequential sputum samples provided by 12 patients with cystic fibrosis and results of subsequent strain identification by molecular typing methods. All isolates identified to the strain-level were included in the final analyses $(n=2055)$

\section{Results}

Baseline patient characteristics are summarised in Table 1 (individual data, Additional file 2: Table S1 and Additional file 3: Table S2). All patients had advanced lung disease, had been chronically infected with $P$. aeruginosa for several years, and with one exception were taking maintenance oral azithromycin with inhaled anti-pseudomonal antibiotics prior to the exacerbation episode. Between-group differences at baseline were not statistically significant. A limited numbers of bacterial co-pathogens were isolated from sputum samples patients at baseline and within prior 3 months (4 patients with methicillin-susceptible Staph. aureus), During the period of exacerbation treatment, all patients received combination intravenous anti-pseudomonal antibiotics and continued taking azithromycin. Inhaled antibiotics were ceased. The median (range) period from

Table 1 Characteristics of the 12 patients with single-strain and mixed-strain infections

\begin{tabular}{|c|c|c|c|}
\hline Variable & Single-strain infection $(n=7)$ & Mixed-strain infection $(n=5)$ & All ${ }^{\mathrm{a}}(n=12)$ \\
\hline Age, mean years (SD) & $33.1(11.2)$ & $30.4(6.1)$ & $32.0(9.2)$ \\
\hline Gender, Male, number (\%) & $5(71.4)$ & $4(80.0)$ & $9(75.0)$ \\
\hline P.Phe508del heterozygous, number (\%) & $5(71.4)$ & $1(20.0)$ & $6(50.0)$ \\
\hline P.Phe508del homozygous, number (\%) & $2(28.6)$ & $4(80.0)$ & $6(50.0)$ \\
\hline $\mathrm{FEV}_{1} \%$ predicted, mean (SD) & $45.9(20.8)$ & $45.6(15.3)$ & $45.8(17.9)$ \\
\hline BMl, mean kg/m² (SD) & $23.7(6.7)$ & $21.6(3.3)$ & $22.9(5.4)$ \\
\hline $\begin{array}{l}\text { Chronic Pseudomonas aeruginosa infection } \\
>10 \text {-years, number (\%) }\end{array}$ & $6(85.7)$ & $5(100)$ & $11(91.7)$ \\
\hline \multicolumn{4}{|l|}{ Clinical care in previous year, mean (SD) } \\
\hline Number of hospital admissions & $2.1(2.0)$ & $4.8(2.9)$ & $3.3(2.7)$ \\
\hline Number of inpatient days & $29.1(30.0)$ & $69.0(37.1)$ & $45.8(37.6)$ \\
\hline Number of outpatient clinic visits & $11.6(8.7)$ & $16.2(3.0)$ & $13.6(7.1)$ \\
\hline \multicolumn{4}{|l|}{$\begin{array}{l}\text { Regular treatments prescribed in previous } \\
\text { year, number }(\%)\end{array}$} \\
\hline Oral azithromycin & $7(100)$ & $5(100)$ & $12(100)$ \\
\hline Inhaled colistin & $3(42.9)$ & $3(60.0)$ & $6(50.0)$ \\
\hline Inhaled tobramycin & $6(85.7)$ & $4(80.0)$ & $10(83.3)$ \\
\hline Diabetes, number (\%) & $1(14.3)$ & $1(20.0)$ & $2(16.7)$ \\
\hline
\end{tabular}

Abbreviations: BMI body mass index, FEV1\% predicted, forced expiratory volume in the first second percentage predicted; SD standard deviation ${ }^{a}$ No patients were diagnosed with CF-related liver disease 
completion of intravenous antibiotics and follow-up visit was $42(13-119)$ days.

\section{Pseudomonas aeruginosa total viable count}

For all patients, the geometric mean $P$. aeruginosa total viable count was similar across the four consecutive time-points of sputum collection (start-of-treatment, $6.3 \times 10^{7}$ colony-forming units $(\mathrm{CFU}) / \mathrm{mL}$; during treatment, $1.8 \times 10^{7} \mathrm{CFU} / \mathrm{mL}$; end-of-treatment, $1.4 \times 10^{8}$ $\mathrm{CFU} / \mathrm{mL}$; follow-up, $1.0 \times 10^{8} \mathrm{CFU} / \mathrm{mL}$ ) with no statistically significant change over time $(P=0.1$, repeated measures ANOVA; Fig. 2).

\section{Genotyping}

The 12 study participants provided 45 sputum samples (Additional file 4: Figure S1), from which 2160 isolates were selected for genotyping (Fig. 1). Of the 2055 isolates genotyped successfully, 1117 (54.4\%) were AUST02 strains, while the remaining isolates comprised other commonly shared Australian strains (AUST-01, $n=2$, $0.1 \%$; AUST-06, $n=752$, 36.6\%; AUST-07, $n=94,4.6 \%$; AUST-11, $n=88,4.3 \%)[13,22]$, with only two isolates $(0.1 \%)$ representing unique strains (Fig. 1). Although allele-specific PCR of a sweep of colonies confirmed all 12 patients harboured AUST-02 at study entry, this strain was not detected amongst any of the individual isolates selected from two patients at each of the four separate time-points; one patient (P12) was positive for AUST-06 only and another (P7) for AUST-01 and AUST-06 (Fig. 3).

\section{Mixed-strain dynamics}

Based on relative abundances of $P$. aeruginosa strains in each sample, patients were categorised into two groups: those with a (1) "single strain infection" (P3, P7, P8, P9, $\mathrm{P} 10, \mathrm{P} 11, \mathrm{P} 12)$ since a single strain comprised $>90 \%$ relative abundance in all samples tested, and (2) "mixedstrain infection" (P1, P2, P4, P5, P6) where various mixtures of strains (at $<90 \%$ relative abundance) were observed (Fig. 3).

A significant temporal variation in the $P$. aeruginosa population composition was found for the five patients with mixed-strain infections $\left(\chi^{2}=56.3, P<0.001\right)$. A gradual reduction in the mean relative abundance $(95 \%$ confidence interval) of AUST-02 was detected in sequential sputum samples compared to the start-oftreatment sample [during treatment, $-20.7 \%(-58.4$ to 17.0\%); end-of-treatment, $-25.4 \% \quad(-46.7$ to $-4.1 \%)$; follow-up, $-34.6 \%$ (-77.2 to $8.1 \%)$ ]. Concurrently, the increased relative abundance of 'other' strains detected

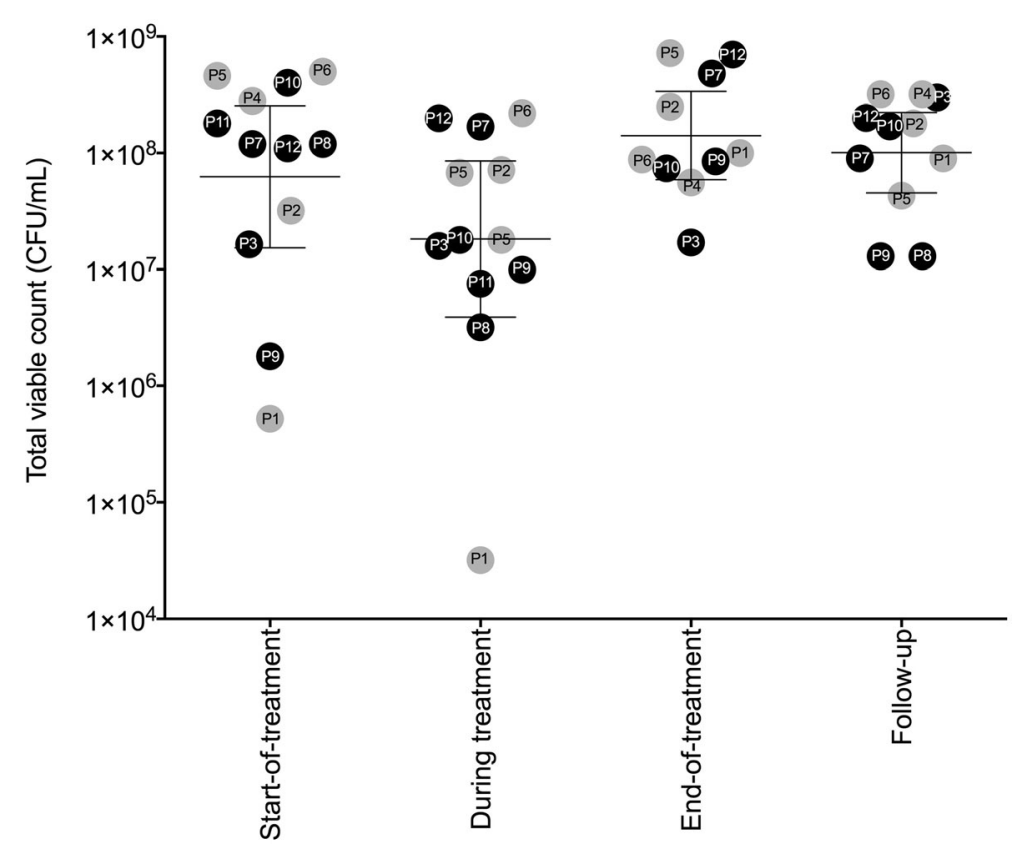

Fig. 2 Geometric means (with 95\% confidence intervals) of total viable counts expressed as colony-forming units (CFU)/mL of Pseudomonas aeruginosa in sputum samples collected from adult patients with cystic fibrosis at start-of-treatment, during treatment and end-of-treatment timepoints for intravenous antibiotic therapy of a pulmonary exacerbation, and at follow-up. Single-strain infection, black circle; mixed-strain infection, grey circle. There was no statistically significant difference between time-points of sputum collection and total viable counts $(P=0.1$, repeated measures ANOVA; based on log-transformed CFU/mL from 10 patients, who each provided four sputum samples). There was also no statistically significant difference between time-points of sputum collection and total viable counts of those with single-strain infections $(P=0.5$, repeated measures ANOVA; based on log-transformed CFU/mL from 5 patients, who each provided four sputum samples) or mixed-strain infections ( $P=0.2$, repeated measures ANOVA; based on log-transformed $\mathrm{CFU} / \mathrm{mL}$ from 5 patients) 


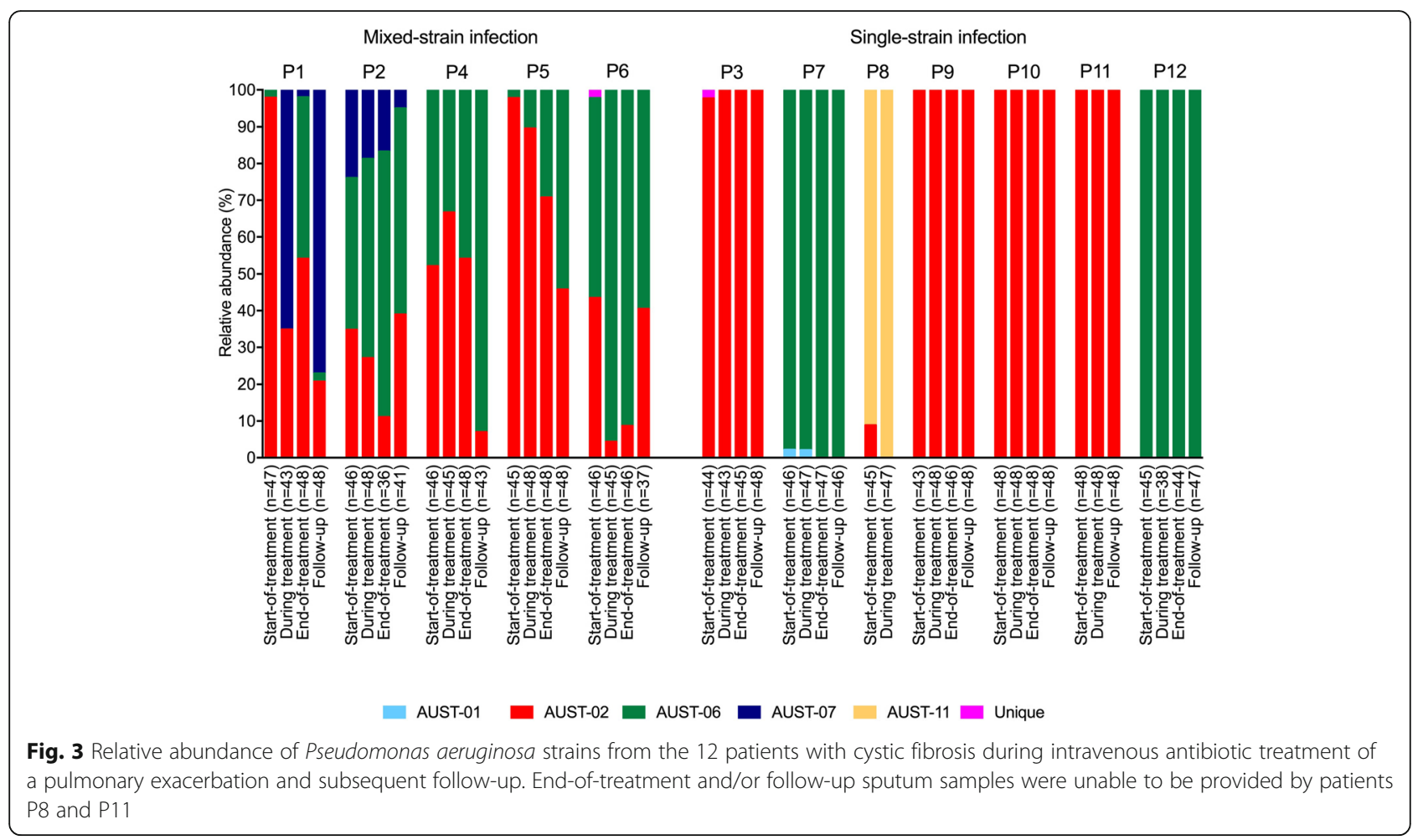

compared to the start-of-treatment was primarily due to a surge in AUST-06 richness at all time-points [during treatment, $+9.1 \%(-16.7$ to $34.9 \%)$; end-of-treatment, $+26.9 \%$ (5.7 to $48.1 \%)$; follow-up, $+23.4 \% \quad(-6.0$ to 52.8\%)]. AUST-02 and AUST-06 temporal dynamics are summarised in Additional file 6: Figure S2.

\section{Phenotypic traits}

Overall, 186 isolates from patient P1 (6/192 isolates were excluded as they were not genotyped successfully) underwent in-vitro assessment of adaptive phenotypic traits (Additional file 5: Table S3). A statistical association was detected between strain (AUST-02, AUST-06, AUST-07) and susceptibility category for $7 / 11$ antibiotics with an equal or higher proportion of AUST-02 isolates categorised as non-susceptible to these seven antibiotics compared to AUST-06 or AUST-07 ( $\chi^{2}$ [range] = 26.0-108.9; $P<0.001$, Additional file 7: Figure S3a). AUST-02 was also more often auxotrophic (92\% isolates) compared to AUST-06 (35\% isolates) and AUST-07 (2\% isolates) $\left(\chi^{2}=131.7 ; P<0.001\right.$, Additional file 7: Figure S3b). Defining phenotypic traits of AUST-06 and AUST-07 were mucoidy $\left(87 \%\right.$ of isolates; $X^{2}=134.9 ; P<0.001$, Additional file 7: Figure S3b) and colony surface iridescent sheen $\left(86 \%\right.$ of isolates; $X^{2}=108.0 ; P<0.001$, Additional file 7: Figure S3b), respectively. Pyocyanin production was similar between all strains (Additional file 7: Figure S3b).

\section{Clinical response to antibiotic treatment}

Despite similar baseline $\mathrm{FEV}_{1} \%$ values and other between-group variables not being significantly different, patients harbouring mixed strains had more long-term treatment requirements than those with single strain infection (Table 1). During treatment of the exacerbation, there was a greater improvement in $\mathrm{FEV}_{1} \%$ predicted in patients with single-strain infection [median change (range): $7.2 \%$ (3.1 to $15.8 \%$ ); $P=0.02$; Wilcoxon signedrank test; Table 2a] compared to those with a mixedstrain infection [median change (range): $2.4 \%$ (0 to $14.5 \%) ; P=0.07$; Wilcoxon signed-rank test; Table 2a), despite the median $\mathrm{FEV}_{1} \%$ predicted being similar between both groups at start-of-treatment [single-strain infection: $31.2 \%$ (range: 20.2 to $84.0 \%$ ); mixed-strain infection: $41.7 \%$ (Range: 30.3 to $63.1 \%$ ); $P=0.5$, Man$\mathrm{n}$-Whitney $\mathrm{U}$ test]. Furthermore, mean CRP was higher in those with single-strain $(33.2 \mathrm{mmol} / \mathrm{L})$ compared to mixed-strain infection $(10.3 \mathrm{mmol} / \mathrm{L})$ at start-oftreatment, albeit not significantly $(P=0.08$, Welch's t-test). After their exacerbation was treated, patients with mixed-strain infections had a shorter time to next admission for exacerbation treatment compared to those with single-strains, but this also did not reach statistical significance (Table $2 \mathrm{~b}$ ).

\section{Discussion}

Shared $P$. aeruginosa strain dynamics during and after antibiotic treatment of a pulmonary exacerbation in 


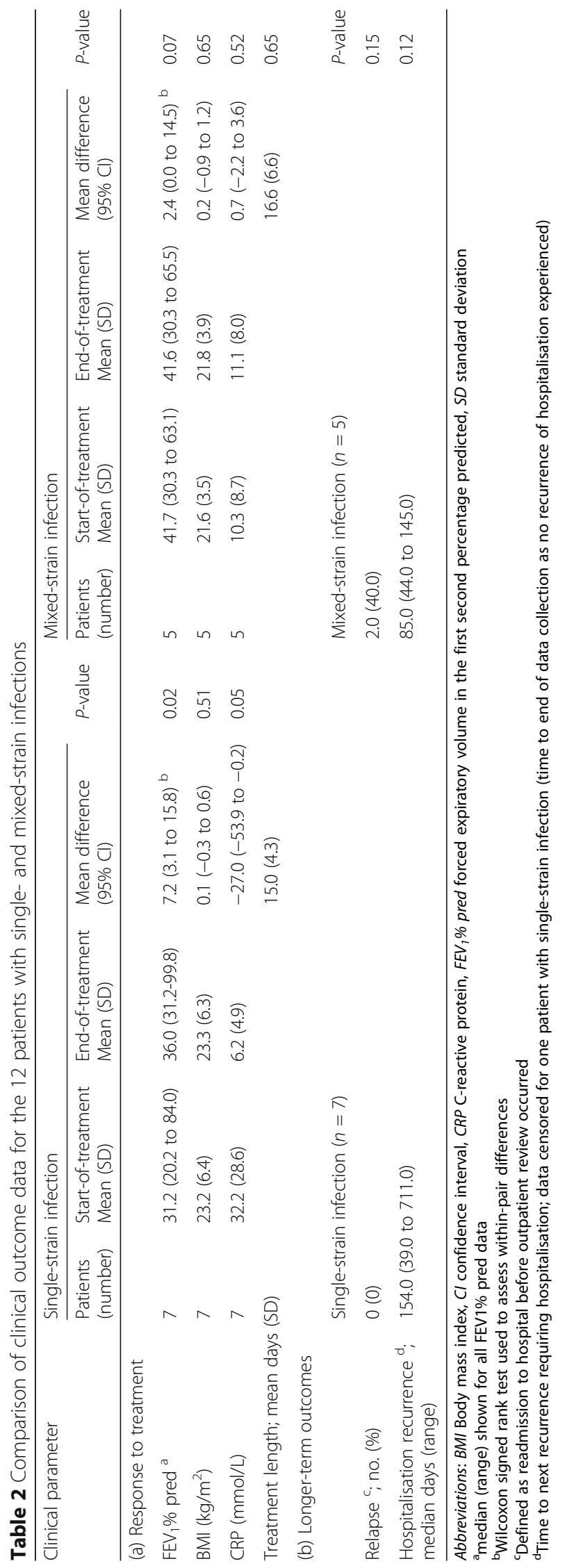


chronically infected CF patients were investigated by combining in-depth culture and genotyping techniques. Interestingly, this Australian study found that a substantial number of CF patients showed mixed-strain infection, which contrasts with the extensive experience in the UK where similar studies of the Liverpool Epidemic Strain (LES) consistently demonstrated that CF patients have chronic infection with a single strain, which has diverged into multiple co-existing sublineages in the airways $[11,26]$. This disparity is possibly related to differences in patient segregation practices between study centres.

Patients with mixed-strain infection showed significant changes in strain composition throughout the entire exacerbation episode and follow-up period, with others typically maintaining the same strain. Almost all other non-AUST-02 isolates (>99\%) identified comprised previously recognised Australian shared strains, suggesting that people infected with one shared strain are more likely to be co-infected with other shared strains than unique strains. Alternatively, it can be argued that patients with advanced lung disease spending greater time in hospital are more likely to be exposed to one or more shared strains [18].

Single-strain infection with LES was previously shown to be associated with increased treatment requirements [27]. Our current study provides some evidence that infection with multiple shared strains is associated with greater morbidity than single-strain infection in Australia. In the year before recruitment and the months following their exacerbation episodes, those with mixedstrain infections had more treatment requirements than those with predominant single-strain infection, although this difference did not reach statistical significance. Further studies involving larger patient numbers and multiple centres are needed to confirm this observation. The data also potentially suggest that short-term intravenous antibiotic courses might not be a driver of the evolution of single strains dominating infection in CF patients. However, given that seven patients harboured a single-strain infection throughout the study period, longitudinal studies are required to determine if one shared strain displaces other(s) in mixed-strain infections as suggested by our observations and reported occasionally by others [15, 28, 29].

Patients with single-strain infections had a higher start-of-treatment CRP with improved lung function observed during treatment of the exacerbation. In contrast, although numbers were limited, those with mixed-strain infection had less evidence of an acute inflammatory response at the start-of-treatment and no significant change in lung function was observed. This further highlights the complexity of defining CF exacerbations and determining the timing and duration of antibiotic therapy, particularly in those with advanced lung disease, who now live longer than previously [30].

Although not statistically significant, we observed a transient decrease in the total $P$. aeruginosa load during the first 6-9 days of intravenous antibiotic treatment, which was reversed by the end of treatment. Similar transient effects have been previously described [31]. However, selection and clonal expansion of a $P$. aeruginosa population with a specific genotype or particular phenotypic traits may underpin pulmonary exacerbations [11, 15, 32, 33]. Whilst we cannot assign causality of exacerbation here, our in-depth sampling approach enabled the first characterisation of the relative abundance and dynamic nature of mixed strains, which cannot be determined if only a few isolates per sample are genotyped [13-15]. We found that the relative abundance of AUST-02 declined during anti-pseudomonal treatment [34]. At the same time, the relative abundance of other shared strains increased, suggesting they were under positive selection pressure. This was true especially for AUST-06, which was identified almost exclusively in Queensland previously and was the second most common shared strain identified here [13]. Whilst previous studies have shown within-host microevolution leads to co-existing sublineages of single $P$. aeruginosa strains emerging over months-to-years [26, 35-37], this study demonstrates rapid multi-strain turnover in mixed-strain infections within-host during antibiotic treatment of pulmonary exacerbations.

It is difficult to explain the observed temporal dynamics of mixed-strain infection, particularly when considering the antibiotic susceptibility profiles of strains from a single patient treated with meropenem, aztreonam and tobramycin. Even though the AUST-02 relative abundance decreased between start-of-treatment and end-oftreatment, this strain exhibited greater or equal in-vitro resistance to these antibiotics than the AUST-06 and AUST-07 strains that emerged in sputum during treatment. These unexpected results emphasise the poor correlation between in-vitro susceptibility testing and in-vivo response [38-40] and suggest some strains harbour alternative mechanisms, such as alginate overproduction and adaptive resistance that enable persistence despite aggressive antibiotic treatment of exacerbations [11, 41-45]. In addition, other virulence determinants may impact the host inflammatory response and co-infection with other CF pathogens [46-48]. Such factors could potentially favor the selection of AUST-06 during intravenous antibiotic treatment. Furthermore, phenotypic traits could generally be assigned to a particular strain type, but more isolates are required to confirm this observation and relate findings to the clinical course $[11,49]$.

While more than 2000 P. aeruginosa isolates were genotyped, the results must be interpreted with caution 
given the small number of patients attending a single centre involved. A further limitation is that sputum was not collected during clinical stability, before the onset of the exacerbation. Therefore, the proportions of strains at clinical stability before and after an exacerbation could not be compared. Sputum may also not be ideal for inferring the overall airway $P$. aeruginosa load or population composition as it may not represent all lung compartments, and whilst bronchoscopic sampling enables collection of regional $P$. aeruginosa populations, this method is too invasive for routine use [50]. Fluctuations in the population composition might represent regional changes in $P$. aeruginosa airway density and local micro-environmental conditions, altered mucus volume or variations in sputum sampling within the lung [37]. The patients had advanced lung disease and were treated with different antibiotic regimens, which may also affect the dynamics and evolution of mixed-strain infections. Furthermore, despite confirming AUST-02 by culture sweep at study entry, using a random culture approach, in two patients who had previously had chronic AUST-02 infection, AUST-02 was not subsequently identified in the genotyping of 48 randomly selected colonies, and only AUST-06 and AUST-01 were detected. It is possible AUST-02 might have constituted a minority of the $P$. aeruginosa population in these cases at the time of study recruitment, and therefore, were not selected because of limitations in sampling.

Further work will now be conducted to assess the relative abundance of shared strains directly in sputum using high-resolution molecular approaches and with larger study populations across a range of disease severities and other CF centres to validate the findings. This will extend to investigating intra-strain diversity and temporal dynamics, including an AUST-02 strain subtype (M3 L7) that we described recently [51].

This exploratory study provides novel data characterising the temporal dynamics of a $P$. aeruginosa mixedstrain population during and after intravenous antibiotic treatment of exacerbations. Various commonly shared strains from throughout Australia, alone or in combination, were identified in individual patients. Together, the data show the rapidly changing strain heterogeneity of pulmonary exacerbations, raising further questions over whether acquiring shared $P$. aeruginosa strains is a marker or cause of more advanced CF lung disease. Ultimately, the much-needed answers to these questions will assist with refining treatments and existing infection control policies within CF centres.

\section{Conclusions}

Within CF airways, multiple co-existing sublineages evolve gradually from a single dominant $P$. aeruginosa strain overtime. Combining in-depth sputum culture and genotyping techniques, we now also show that patients harbouring the AUST-02 strain may have other unrelated, but commonly shared, $P$. aeruginosa strains whose relative abundance with one another may change rapidly in a sustained and unpredictable manner during antibiotic treatment.

\section{Additional files}

\begin{abstract}
Additional file 1: Supplementary Methods. (DOCX $17 \mathrm{~kb}$ )
Additional file 2: Table S1. Characteristics of individual patients before the exacerbation episode. (DOCX $18 \mathrm{~kb}$ )
\end{abstract}

Additional file 3: Table S2. Treatment received during the exacerbation and clinical outcome data. (DOCX $18 \mathrm{~kb}$ )

Additional file 4: Figure S1. Timeline of sputa collection from 12 patients. (DOCX $48 \mathrm{~kb}$ )

Additional file 5: Table S3. Results of phenotypic testing of strains (AUST-02, AUST-06, AUST-07) isolated from each sputum sample provided by patient 1. (DOCX $27 \mathrm{~kb})$

Additional file 6: Figure S2. Temporal dynamics of the total proportions of AUST-02 and AUST-06 shared Pseudomonas aeruginosa strains detected during the course of intravenous antibiotic treatment of an exacerbation and subsequent follow-up for patients with mixed-strain infections. (DOCX 1092 kb)

Additional file 7: Figure S3. Characterization of adaptive phenotypic traits of within-patient (P1) mixed-strain infection. (DOCX 1101 kb)

\section{Abbreviations}

ANOVA: Analysis of variance; BMl: Body mass index; CF: Cystic fibrosis; CFTR: Cystic fibrosis transmembrane conductance regulator; CFU: Colonyforming unit; CRP: C-reactive protein; FEV $\%$ : Forced expiratory volume in the first second percentage predicted; PCR: Polymerase chain reaction; SNP: Single nucleotide polymorphism

\section{Acknowledgments}

Not applicable.

\section{Funding}

This study was funded by grants from the National Health and Medical Research Council (NHMRC) (\#455919), Children's Health Foundation Queensland (\#50007), Queensland Health Office of Health and Medical Research, Australian Cystic Fibrosis Research Trust (\#2009-06), The Prince Charles Hospital Foundation, Rotary Australia and CF Foundation

Therapeutics (USA); NHMRC Medical and Dental Postgraduate Scholarship (APP1017517) and Australian Cystic Fibrosis Research Trust Scholarship to AST, NHMRC Early Career Fellowship (\#1088448) and ERS-EU RESPIRE2 Marie Skłodowska-Curie Postdoctoral Research Fellowship (\#4571-2013) to TJK, NHMRC Career Development Fellowship (APP1054129) to DMW, Australian Postgraduate Award PhD Scholarship (\#2127932) and an Australian Cystic Fibrosis Research Trust Postgraduate Scholarship to KAR, Queensland Health, Health Research Fellowship (QCOS013795) to SCB, Shelley Shephard Memorial Scholarship to LJS.

None of the above funding bodies has any role in the study design; data collection, analysis and interpretation; or writing the manuscript.

\section{Availability of data and materials}

The datasets generated during and/or analysed during the current study are available from the corresponding author on reasonable request.

\section{Authors' contributions}

DMW and SCB jointly supervised the study. AST, CB and MS performed experiments. AST and LJS analyzed the data. AST and LJS wrote the initial manuscript. DMW, SCB, KG, TJK and KAR helped to interpret the data, write the manuscript and provide revisions. All authors reviewed and revised the final manuscript. All authors read and approved the final manuscript. 


\section{Ethics approval and consent to participate}

The Prince Charles Hospital Human and Research Ethics Committee, Metro North Hospital and Health Service, Brisbane, Queensland, Australia approved the study (HREC/13/QPCH/127) and all participants provided written, informed consent.

\section{Consent for publication}

Not applicable (no individual level patient data contained within this manuscript).

\section{Competing interests}

DMW reports research funding from SpeeDx Pty Ltd. which has no conflict of interest with current study. AST, LJS, TJK, KAR, CB, MS, KG, SCB declare no competing interests.

\section{Publisher's Note}

Springer Nature remains neutral with regard to jurisdictional claims in published maps and institutional affiliations.

\section{Author details \\ 'School of Medicine, The University of Queensland, Brisbane, QLD, Australia. ${ }^{2}$ Adult Cystic Fibrosis Centre, Department of Thoracic Medicine, The Prince Charles Hospital, Brisbane, QLD, Australia. ${ }^{3}$ Western Australia Adult Cystic Fibrosis Centre, Department of Respiratory Medicine, Sir Charles Gairdner Hospital, Hospital Avenue, Perth, WA 6009, Australia. ${ }^{4}$ Lung Bacteria Group, QIMR Berghofer Medical Research Institute, Brisbane, QLD, Australia. ${ }^{5}$ School of Chemistry and Molecular Biosciences, The University of Queensland, Brisbane, QLD, Australia. ${ }^{6}$ Centre for Experimental Medicine, Queen's University Belfast, Belfast, UK. ${ }^{7}$ Child Health Research Centre, The University of Queensland, Brisbane, QLD, Australia. ${ }^{8}$ UQ Centre for Clinical Research, The University of Queensland, Brisbane, QLD, Australia. ${ }^{9}$ Pathology Queensland, Microbiology Department, Brisbane, QLD, Australia. ${ }^{10}$ Menzies Health Institute Queensland, Griffith University and Gold Coast Health, Gold Coast, QLD, Australia.}

Received: 31 May 2017 Accepted: 25 October 2017

Published online: 02 November 2017

\section{References}

1. Foundation CF. Cystic Fibrosis foundation patient registry 2012 annual data report. Bethesda: Cystic Fibrosis Foundation; 2013.

2. Sanders DB, Bittner RC, Rosenfeld M, Redding GJ, Goss CH. Pulmonary exacerbations are associated with subsequent $\mathrm{FEV}_{1}$ decline in both adults and children with cystic fibrosis. Pediatr Pulmonol. 2011;46(4):393-400.

3. Amadori A, Antonelli A, Balteri I, Schreiber A, Bugiani M, et al. Recurrent exacerbations affect FEV(1) decline in adult patients with cystic fibrosis. Respir Med. 2009;103(3):407-13.

4. Cogen J, Emerson J, Sanders DB, Ren C, Schechter MS, et al. Risk factors for lung function decline in a large cohort of young cystic fibrosis patients. Pediatr Pulmonol. 2015;50(8):763-70.

5. Solem CT, Vera-Llonch M, Liu S, Botteman M, Castiglione B. Impact of pulmonary exacerbations and lung function on generic health-related quality of life in patients with cystic fibrosis. Health Qual Life Outcomes. 2016;14:63.

6. de Boer K, Vandemheen KL, Tullis E, Doucette S, Fergusson D, et al. Exacerbation frequency and clinical outcomes in adult patients with cystic fibrosis. Thorax. 2011;66(8):680-5.

7. Goss CH, Burns JL. Exacerbations in cystic fibrosis. 1: epidemiology and pathogenesis. Thorax. 2007;62(4):360-7.

8. Ramsey BW, Pepe MS, Quan JM, Otto KL, Montgomery AB, et al. Intermittent administration of inhaled tobramycin in patients with cystic fibrosis. Cystic fibrosis inhaled Tobramycin study group. N Engl J Med. 1999;340(1):23-30.

9. Fuchs HJ, Borowitz DS, Christiansen DH, Morris EM, Nash ML, et al. Effect of aerosolized recombinant human DNase on exacerbations of respiratory symptoms and on pulmonary function in patients with cystic fibrosis. The Pulmozyme study group. N Engl J Med. 1994;331(10):637-42.

10. Doring G, Flume P, Heijerman H, Elborn JS, Consensus Study G. Treatment of lung infection in patients with cystic fibrosis: current and future strategies. J Cyst Fibros. 2012;11(6):461-79.
11. Mowat E, Paterson S, Fothergill JL, Wright EA, Ledson MJ, et al. Pseudomonas aeruginosa population diversity and turnover in cystic fibrosis chronic infections. Am J Respir Crit Care Med. 2011;183(12):1674-9.

12. Hilliam Y, Moore MP, Lamont IL, et al. Pseudomonas aeruginosa adaptation and diversification in the non-cystic fibrosis bronchiectasis lung. Eur Respir J Physiol. 2017;49:1602108. https://doi.org/10.1183/13993003.02108-2016.

13. Kidd TJ, Ramsay KA, Hu H, Marks GB, Wainwright CE, et al. Shared Pseudomonas aeruginosa genotypes are common in Australian cystic fibrosis centres. Eur Respir J Physiology. 2013;41(5):1091-100.

14. Aaron SD, Vandemheen KL, Ramotar K, Giesbrecht-Lewis T, Tullis E, et al. Infection with transmissible strains of Pseudomonas aeruginosa and clinical outcomes in adults with cystic fibrosis. JAMA. 2010;304(19):2145-53.

15. Aaron SD, Ramotar K, Ferris W, Vandemheen K, Saginur R, et al. Adult cystic fibrosis exacerbations and new strains of Pseudomonas aeruginosa. Am J Respir Crit Care Med. 2004;169(7):811-5.

16. Lee TW, Brownlee KG, Conway SP, Denton M, Littlewood JM. Evaluation of a new definition for chronic Pseudomonas aeruginosa infection in cystic fibrosis patients. J Cyst Fibros. 2003;2(1):29-34.

17. Ramsay KA, Sandhu H, Geake JB, Ballard E, O'Rourke P, et al. The changing prevalence of pulmonary infection in adults with cystic fibrosis: a longitudinal analysis. J Cyst Fibros. 2017;16(1):70-7.

18. Kidd TJ, Magalhaes RJ, Paynter S, Bell SC, Group ACI. The social network of cystic fibrosis centre care and shared Pseudomonas aeruginosa strain infection: a cross-sectional analysis. Lancet Respir Med. 2015;3(8):640-50.

19. Hankinson JL, Odencrantz JR, Fedan KB. Spirometric reference values from a sample of the general U.S. population. Am J Respir Crit Care Med. 1999; 159(1):179-87.

20. Kidd TJ, Ramsay KA, Hu H, Bye PT, Elkins MR, et al. Low rates of Pseudomonas aeruginosa misidentification in isolates from cystic fibrosis patients. J Clin Microbiol. 2009;47(5):1503-9.

21. Anuj SN, Whiley DM, Kidd TJ, Ramsay KA, Bell SC, et al. Rapid singlenucleotide polymorphism-based identification of clonal Pseudomonas aeruginosa isolates from patients with cystic fibrosis by the use of realtime PCR and high-resolution melting curve analysis. Clin Microbiol Infect. 2011;17(9):1403-8.

22. Syrmis MW, Kidd TJ, Moser RJ, Ramsay KA, Gibson KM, et al. A comparison of two informative SNP-based strategies for typing Pseudomonas aeruginosa isolates from patients with cystic fibrosis. BMC Infect Dis. 2014; 14(1):307.

23. CLSI. Clinical and laboratory standards institute. Performance standards for antimicrobial susceptibility testing; twenty-third informational supplement. CLSI document M100-S23. Wayne: Clinical and Laboratory Standards Institute; 2013. p. 2013.

24. Fyfe JA, Govan JR. Alginate synthesis in mucoid Pseudomonas aeruginosa: a chromosomal locus involved in control. J Gen Microbiol. 1980;119(2):443-50.

25. D'Argenio DA, Wu M, Hoffman LR, Kulasekara HD, Deziel E, et al. Growth phenotypes of Pseudomonas aeruginosa lasR mutants adapted to the airways of cystic fibrosis patients. Mol Microbiol. 2007;64(2):512-33.

26. Williams D, Evans B, Haldenby S, Walshaw MJ, Brockhurst MA, et al. Divergent, coexisting Pseudomonas aeruginosa lineages in chronic cystic fibrosis lung infections. Am J Respir Crit Care Med. 2015;191(7):775-85.

27. Jones AM, Dodd ME, Doherty CJ, Govan JR, Webb AK. Increased treatment requirements of patients with cystic fibrosis who harbour a highly transmissible strain of Pseudomonas aeruginosa. Thorax. 2002;57(11):924-5.

28. Duong J, Booth SC, McCartney NK, Rabin HR, Parkins MD, et al. Phenotypic and genotypic comparison of epidemic and non-epidemic strains of Pseudomonas aeruginosa from individuals with cystic fibrosis. PLoS One. 2015;10(11):e0143466.

29. Griffiths AL, Jamsen K, Carlin JB, Grimwood K, Carzino R, et al. Effects of segregation on an epidemic Pseudomonas aeruginosa strain in a cystic fibrosis clinic. Am J Respir Crit Care Med. 2005;171(9):1020-5.

30. George PM, Banya W, Pareek N, Bilton D, Cullinan P, et al. Improved survival at low lung function in cystic fibrosis: cohort study from 1990 to 2007. BMJ. 2011;342:d1008

31. Smith DJ, Badrick AC, Zakrzewski M, Krause L, Bell SC, et al. Pyrosequencing reveals transient cystic fibrosis lung microbiome changes with intravenous antibiotics. Eur Respir J. 2014;44(4):922-30.

32. Price KE, Hampton TH, Gifford AH, Dolben EL, Hogan DA, et al. Unique microbial communities persist in individual cystic fibrosis patients throughout a clinical exacerbation. Microbiome. 2013;1(1):27. 
33. Diaz Caballero J, Clark ST, Coburn B, Zhang Y, Wang PW, et al. Selective sweeps and parallel Pathoadaptation drive Pseudomonas aeruginosa evolution in the cystic fibrosis lung. MBio. 2015;6(5)

34. Workentine ML, Sibley CD, Glezerson B, Purighalla S, Norgaard-Gron JC, et al. Phenotypic heterogeneity of Pseudomonas aeruginosa populations in a cystic fibrosis patient. PLoS One. 2013;8(4):e60225.

35. Cramer N, Klockgether J, Wrasman K, Schmidt M, Davenport CF, et al. Microevolution of the major common Pseudomonas aeruginosa clones $C$ and PA14 in cystic fibrosis lungs. Environ Microbiol. 2011;13(7):1690-704.

36. Marvig RL, Sommer LM, Molin S, Johansen HK. Convergent evolution and adaptation of Pseudomonas aeruginosa within patients with cystic fibrosis. Nat Genet. 2015;47(1):57-64.

37. Jorth P, Staudinger BJ, Wu X, Hisert KB, Hayden H, et al. Regional isolation drives bacterial diversification within cystic fibrosis lungs. Cell Host Microbe. 2015:18(3):307-19.

38. Smith AL, Fiel SB, Mayer-Hamblett N, Ramsey B, Burns JL. Susceptibility testing of Pseudomonas aeruginosa isolates and clinical response to parenteral antibiotic administration: lack of association in cystic fibrosis. Chest. 2003;123(5):1495-502.

39. Hurley MN, Ariff AH, Bertenshaw C, Bhatt J, Smyth AR. Results of antibiotic susceptibility testing do not influence clinical outcome in children with cystic fibrosis. J Cyst Fibro. 2012;11(4):288-92.

40. Parkins MD, Rendall JC, Elborn JS. Incidence and risk factors for pulmonary exacerbation treatment failures in patients with cystic fibrosis chronically infected with Pseudomonas aeruginosa. Chest. 2012;141(2):485-93.

41. Fothergill JL, Mowat E, Ledson MJ, Walshaw MJ, Winstanley C. Fluctuations in phenotypes and genotypes within populations of Pseudomonas aeruginosa in the cystic fibrosis lung during pulmonary exacerbations. J Med Microbiol. 2010;59(Pt 4):472-81.

42. Hatch RA, Schiller NL. Alginate lyase promotes diffusion of aminoglycosides through the extracellular polysaccharide of mucoid Pseudomonas aeruginosa. Antimicrob Agents Chemother. 1998;42(4):974-7.

43. Parad RB, Gerard CJ, Zurakowski D, Nichols DP, Pier GB. Pulmonary outcome in cystic fibrosis is influenced primarily by mucoid Pseudomonas aeruginosa infection and immune status and only modestly by genotype. Infect Immun. 1999;67(9):4744-50.

44. Barclay ML, Begg EJ, Chambers ST, Thornley PE, Pattemore PK, et al. Adaptive resistance to tobramycin in Pseudomonas aeruginosa lung infection in cystic fibrosis. J Antimicrob Chemother. 1996;37(6):1155-64.

45. Boucher JC, Yu H, Mudd MH, Deretic V. Mucoid Pseudomonas aeruginosa in cystic fibrosis: characterization of muc mutations in clinical isolates and analysis of clearance in a mouse model of respiratory infection. Infect Immun. 1997;65(9):3838-46.

46. Goolaerts A, Lafargue M, Song Y, Miyazawa B, Arjomandi M, et al. PAl-1 is an essential component of the pulmonary host response during Pseudomonas aeruginosa pneumonia in mice. Thorax. 2011;66(9):788-96.

47. de Lima CD, Calegari-Silva TC, Pereira RM, Santos SA, Lopes UG, et al. Exol activates NF-kappaB and increases IL-8/KC secretion during Pseudomonas aeruginosa infection. PLoS One. 2012;7(7):e41772.

48. Baldan R, Cigana C, Testa F, Bianconi I, De Simone M, et al. Adaptation of Pseudomonas aeruginosa in cystic fibrosis airways influences virulence of Staphylococcus aureus in vitro and murine models of co-infection. PLoS One. 2014;9(3):e89614.

49. Nixon GM, Armstrong DS, Carzino R, Carlin JB, Olinsky A, et al. Clinical outcome after early Pseudomonas aeruginosa infection in cystic fibrosis. J Pediatr. 2001;138(5):699-704.

50. Aaron SD, Kottachchi D, Ferris WJ, Vandemheen KL, St Denis ML, et al. Sputum versus bronchoscopy for diagnosis of Pseudomonas aeruginosa biofilms in cystic fibrosis. Eur Respir J Physiology. 2004;24(4):631-7.

51. Tai AS, Bell SC, Kidd TJ, Trembizki E, Buckley C, et al. Genotypic diversity within a single Pseudomonas aeruginosa strain commonly shared by Australian patients with cystic fibrosis. PLoS One. 2015;10(12):e0144022.

\section{Submit your next manuscript to BioMed Central and we will help you at every step:}

- We accept pre-submission inquiries

- Our selector tool helps you to find the most relevant journal

- We provide round the clock customer support

- Convenient online submission

- Thorough peer review

- Inclusion in PubMed and all major indexing services

- Maximum visibility for your research

Submit your manuscript at www.biomedcentral.com/submit
() BioMed Central 